\title{
Diffuse X-ray emission from the planetary nebula NGC 7009^
}

\author{
M. A. Guerrero, R. A. Gruendl, and Y.-H. Chu \\ Astronomy Department, University of Illinois, 1002 W. Green Street, Urbana, IL 61801, USA \\ Received 7 February 2002 / Accepted 1 March 2002

\begin{abstract}
XMM-Newton EPIC observations of the planetary nebula (PN) NGC 7009, the Saturn Nebula, have detected extended X-ray emission in its central cavity. The diffuse X-ray emission must originate in the shocked fast stellar wind. The spectra show that the temperature of the hot gas is $1.8 \times 10^{6} \mathrm{~K}$. The rms density derived from the volume emission measure is a few tens $\mathrm{H}$-atom $\mathrm{cm}^{-3}$. The hot gas does not appear over-pressurized with respect to the nebular shell. NGC 7009 represents an evolutionary stage at which the influence of the hot gas in the PN interior starts to decline due to the diminishing strength of the fast stellar wind and the expansion of the central cavity.
\end{abstract}

Key words. ISM: planetary nebulae: general - ISM: planetary nebulae: individual: NGC 7009 stars: winds, outflows - X-rays: ISM

\section{Introduction}

It has been commonly accepted that the shell structure of a planetary nebula $(\mathrm{PN})$ is formed by the current fast stellar wind sweeping up the previous asymptotic giant branch (AGB) wind (e.g. Kwok 1983). In this interacting-stellar-winds model, a PN will comprise a central cavity filled with shocked fast wind at temperatures of $10^{6}-10^{8} \mathrm{~K}$, a dense shell of swept-up AGB wind at $10^{4} \mathrm{~K}$, and an outer envelope of unperturbed expanding AGB wind. The shocked fast wind has a high temperature but does not emit appreciably in X-rays because its density is low. At the interface between the shocked fast wind and the dense nebular shell, heat conduction lowers the temperature and mass evaporation raises the density of the shocked fast wind, producing optimal conditions for X-ray emission (Weaver et al. 1977). Thus, soft X-ray emission from the shocked fast wind in a $\mathrm{PN}$ interior is expected to peak near the inner wall of the nebular shell.

The ROSAT X-ray satellite had been used to search for diffuse X-ray emission from $\mathrm{PNe}$ with limited success (Guerrero et al. 2000). It is only recently that the Chandra $X$-ray Observatory has unambiguously detected diffuse $\mathrm{X}$ ray emission in the central cavities of three young PNe: BD +30³639 (Kastner et al. 2000), NGC 6543 (Chu et al. 2001), and NGC 7027 (Kastner et al. 2001). In the case of NGC 6543 where the X-ray emission is well-resolved, a clear limb-brightening is observed. The plasma temperatures implied by their X-ray spectra are a few $10^{6} \mathrm{~K}$.

Send offprint requests to: M. A. Guerrero,

e-mail: mar@astro.uiuc.edu

* Based on observations obtained with XMM-Newton, an ESA science mission with instruments and contributions directly funded by ESA Member States and NASA.
Chandra observations of the evolved PN NGC 7293 (the Helix Nebula), on the other hand, does not show any diffuse X-ray emission (Guerrero et al. 2001). These results suggest that the hot gas content of a PN diminishes as the $\mathrm{PN}$ ages. To confirm this hypothesis, $\mathrm{PNe}$ at intermediate evolutionary stages need to be observed.

We have examined all pointed and serendipitous observations of $\mathrm{PNe}$ in the ROSAT archive and found that NGC 7009, the Saturn Nebula, hosts an X-ray source with spectral characteristics of plasma emission. NGC 7009 is more evolved than the three PNe with diffuse X-ray emission because of its larger nebular size and dynamical age. Consequently, NGC 7009 is an excellent candidate not only for diffuse X-ray emission, but also as a PN at intermediate evolutionary stages. We have obtained X-ray observations of NGC 7009 with the XMM-Newton Observatory, and found that the X-ray emission is indeed extended. This paper reports our analysis of the diffuse X-ray emission from NGC 7009.

\section{Observations}

NGC7009 was observed with the XMM-Newton Observatory in Revolution 255 on 2001 April 30 using the EPIC/MOS1, EPIC/MOS2, and EPIC/pn CCD cameras. The two EPIC/MOS cameras were operated in the Full-Frame Mode for a total exposure time of $39.4 \mathrm{ks}$, while the EPIC/pn camera was operated in the Extended Full-Frame Mode for a total exposure time of $31.6 \mathrm{ks}$. The EPIC/pn observations started about $4 \mathrm{ks}$ later than the EPIC/MOS observations because of a larger overhead required by the EPIC/pn, but both observations concluded at the same time. For all observations, the Medium filter was used. At energies below $1 \mathrm{keV}$, the 


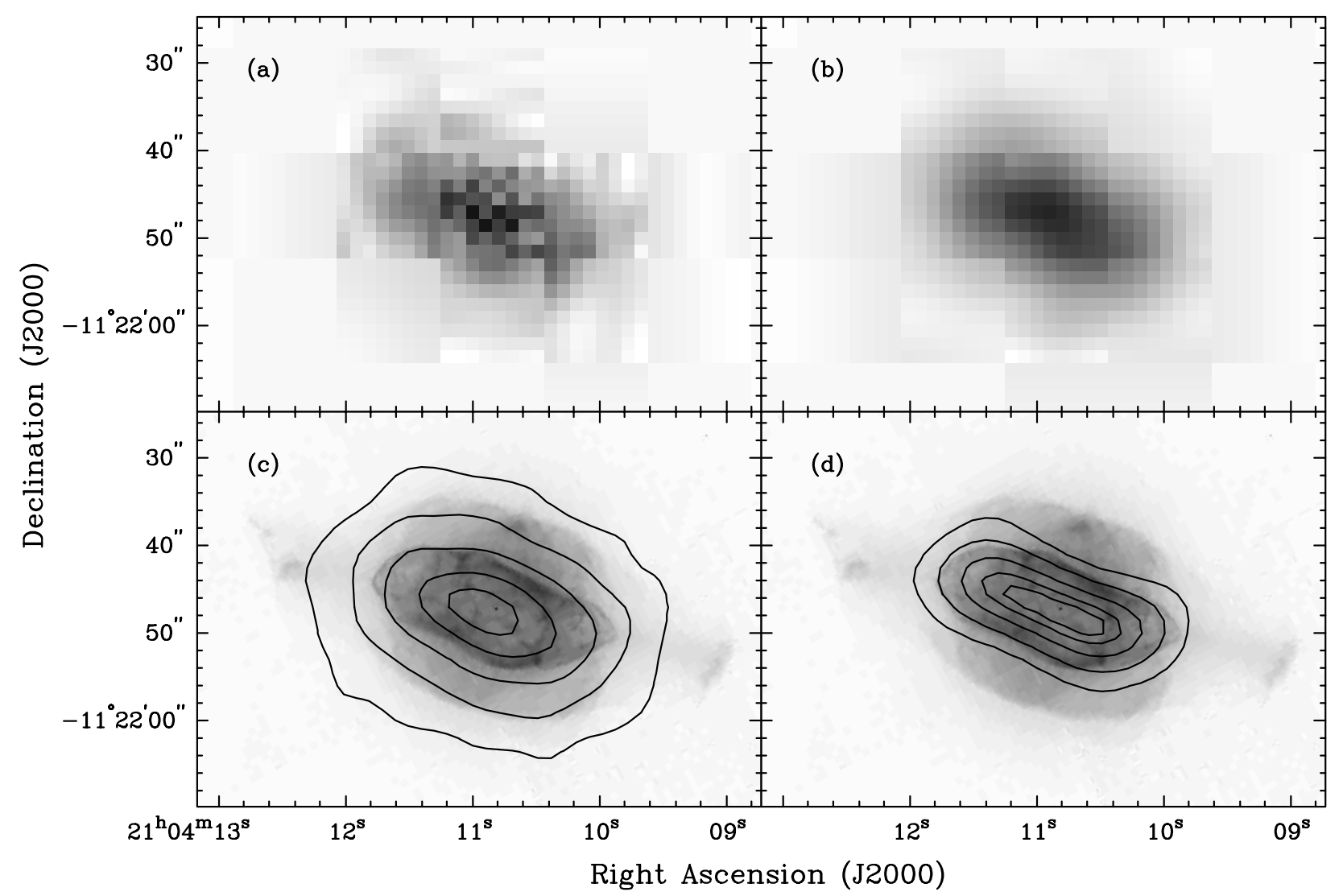

Fig. 1. XMM-Newton EPIC observations of NGC 7009 in the $0.15-1.25 \mathrm{keV}$ band. a) The raw image, b) the adaptively smoothed image, c) X-ray contours from the adaptively smoothed image overlaid on an HST WFPC2 H $\alpha$ image, and d) X-ray contours from PSF-deconvolved EPIC image overlaid on the same $\mathrm{H} \alpha$ image. The contour levels are at 10, 25, 50, 75, and 90\% of the emission peak.

point spread function (PSF) of the EPIC instruments has a small core with $F W H M \sim 6^{\prime \prime}$ but extended wings with a half energy width of $\sim 15^{\prime \prime}$. For the EPIC/MOS and EPIC/pn CCDs, the pixel sizes are 1. . 1 and $4{ }^{\prime \prime} 1$, and the energy resolutions are $\sim 60 \mathrm{eV}$ and $\sim 100 \mathrm{eV}$ at $1 \mathrm{keV}$, respectively (Dahlem 1999; Strüder et al. 2001).

We received the $X M M$ pipeline products which included the raw and processed data of all instruments. These data were further processed using the XMMNewton Science Analysis Software (SAS version 5.2.0) and the calibration files from the Calibration Access Layer available on 2002 January 24.

The event files were further screened to eliminate bad events, such as those due to charged particles, and periods of high background. For the EPIC/MOS observations, only events with CCD patterns 0-12 (similar to $A S C A$ grades 0-4) were selected; for the EPIC/pn observation, only events with CCD pattern 0 (single pixel events) were selected. To assess the background rate, we binned the counts over $25 \mathrm{~s}$ time intervals for each instrument in the 10-12 keV energy range, where the counts were dominated by the background. The time intervals with high background, i.e. count rates $\geq 0.4 \mathrm{cnts} \mathrm{s}^{-1}$ for the EPIC/MOS or $\geq 1.5 \mathrm{cnts} \mathrm{s}^{-1}$ for the EPIC/pn, were discarded. The resulting exposure times are $35.0 \mathrm{ks}, 35.4 \mathrm{ks}$, and $26.2 \mathrm{ks}$ for the EPIC/MOS1, EPIC/MOS2, and EPIC/pn observations, respectively. A total of $480 \pm 25 \mathrm{cnts}, 470 \pm 25 \mathrm{cnts}$, and $1610 \pm 45 \mathrm{cnts}$ from NGC 7009 were detected in the EPIC/MOS1, EPIC/MOS2, and EPIC/pn observations, respectively.

\section{Spatial analysis}

We have analyzed the spatial distribution of the X-ray emission from NGC7009 and compared it to the optical nebula. As the data analysis is fairly complex, we present images at various stages of the processing in Fig. 1. First, the event files from the three EPIC instruments are merged together to construct a raw EPIC image with a pixel size of $1^{\prime \prime} 5$ in the $0.15-1.25 \mathrm{keV}$ energy band (Fig. 1a). As shown in Sect. 4, this energy band includes all photons detected from NGC 7009. The raw EPIC image is then adaptively smoothed using Gaussian profiles with $F W H M$ ranging from $5^{\prime \prime}$ to $17^{\prime \prime}$. A greyscale presentation of this adaptively smoothed EPIC image is shown in Fig. 1b. The contours from this smoothed EPIC image are overplotted on an $\mathrm{H} \alpha$ image of NGC 7009 taken with the Hubble Space Telescope (HST) Wide Field Planetary Camera 2 (WFPC2) and presented in Fig. 1c. Finally, to improve the spatial resolution, this smoothed 

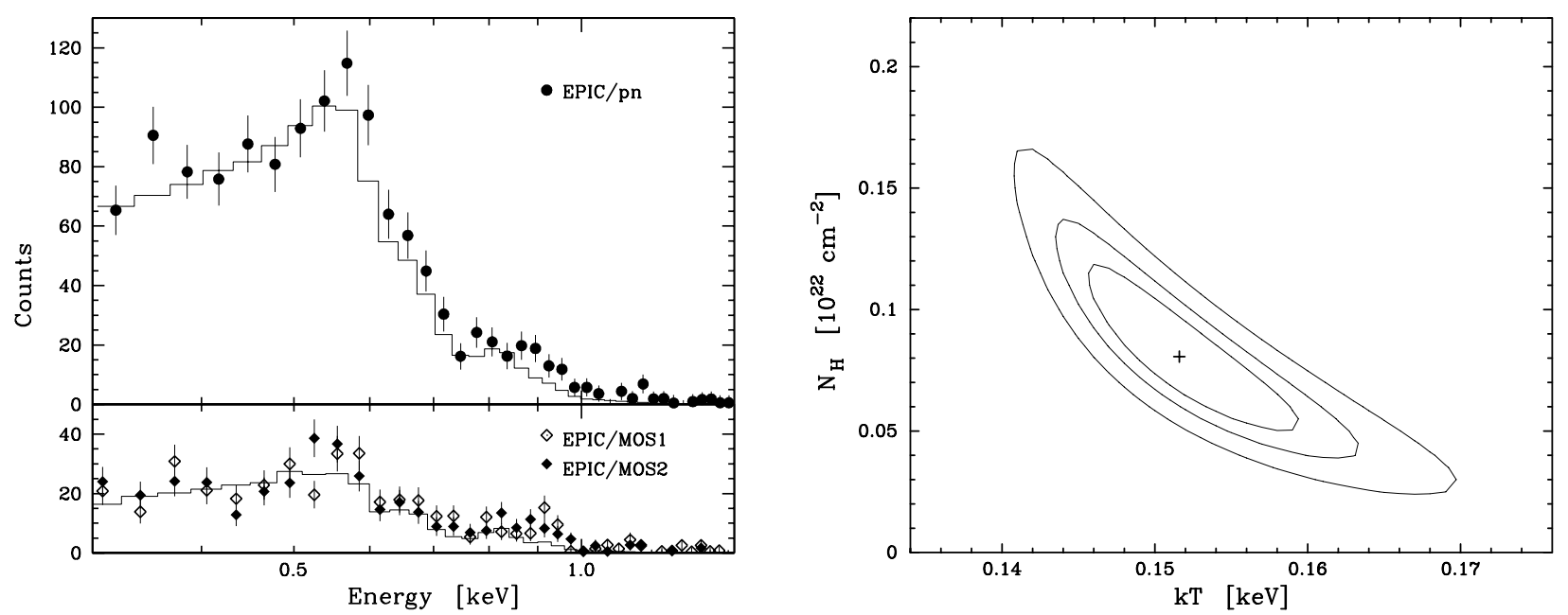

Fig. 2. (left) XMM-Newton EPIC/pn (top), and EPIC/MOS1 and EPIC/MOS2 (bottom) background-substracted spectra of NGC 7009. The spectra are overplotted with the best-fit Raymond-Smith model to the EPIC/pn and the combined EPIC/MOS spectra. Both spectra and models are plotted in $30 \mathrm{eV}$ wide energy bins to preserve the EPIC spectral resolution $(\sim 60-100 \mathrm{eV})$. (right) $\chi^{2}$ grid plot of the spectral fit of the EPIC/pn and the combined EPIC/MOS spectra. The contours represent $68 \%, 90 \%$, and $99 \%$ confidence levels, and the "+" sign corresponds to the parameters of the best-fit model.

EPIC image is deconvolved using the Lucy-Richardson algorithm and a theoretical EPIC PSF provided in the $X M M-N e w t o n$ Calibration Access Layer. The contours extracted from the PSF-deconvolved EPIC image are overplotted on the HST WFPC2 $\mathrm{H} \alpha$ image in Fig. 1d.

The X-ray emission from NGC7009 is clearly extended. Its spatial distribution in the adaptively smoothed EPIC image (Figs. 1b and 1c) can be described by an elliptical Gaussian with $F W H M \sim 23^{\prime \prime} \times 14^{\prime \prime}$ and a major axis along the position angle $\sim 70^{\circ}$. There is an excellent correspondence between the X-ray emission and the nebular shell of NGC 7009. The HST WFPC2 $\mathrm{H} \alpha$ image of NGC 7009 shows a bright $25^{\prime \prime} \times 10^{\prime \prime}$ inner shell surrounded by a $25^{\prime \prime} \times 20^{\prime \prime}$ envelope, with two ansae extending from the tips of the major axes of the inner elliptical shell out to $25^{\prime \prime}$ from the central star. The position and orientation of the $50 \%$ contour of the smoothed EPIC image matches almost perfectly the inner shell of the nebula (Fig. 1c), indicating that the X-ray emission peaks within the central cavity. The PSF-deconvolved EPIC image further shows that the majority of the X-ray emission originates from the central cavity. As the EPIC PSF has extended wings and our PSF-deconvolution is limited by the number of counts and the validity of the theoretical PSF adopted, it is most likely that all diffuse X-ray emission from NGC 7009 is confined within the inner shell, similar to what is observed in $\mathrm{BD}+30^{\circ} 3639$ and NGC 6543. Furthermore, NGC 7009 has a sharp-edged inner shell similar to that of NGC 6543, which implies an expansion driven by the pressure of the hot gas in the central cavity (Chu et al. 2001). Therefore, we suggest that the hot, X-ray-emitting gas in NGC 7009 is also confined within its central cavity, but the detailed distribution of the hot gas cannot be determined from the currently available X-ray observations. In particular, it is not possible to address whether X-ray emission from its central star is present or not.

\section{Spectral analysis}

Spectra of NGC 7009 have been extracted from the event files of the three XMM-Newton EPIC cameras separately because they have different instrumental response functions. A $50^{\prime \prime} \times 40^{\prime \prime}$ elliptical source aperture is used to include all X-ray emission detected from NGC 7009. An elliptical annular region exterior to and $\sim 10$ times as large as the source aperture is used to assess the background level. The background-subtracted spectra of NGC 7009 are shown in Fig. 2 (left).

The EPIC/pn spectrum peaks at $\sim 0.55 \mathrm{keV}$ and drops abruptly to a faint plateau between 0.7 and $1.0 \mathrm{keV}$, but stays essentially flat from $0.5 \mathrm{keV}$ down to $0.3 \mathrm{keV}$. The EPIC/MOS spectra also peak at $\sim 0.55 \mathrm{keV}$, but have smaller numbers of counts because of lower sensitivities. The detailed differences between the EPIC/pn and EPIC/MOS spectra reflect the different instrumental responses among these cameras and the low $S / N$ ratios in the latter spectra. Neither the EPIC/MOS spectra nor the EPIC/pn spectrum shows any detectable emission at energies greater than $1.25 \mathrm{keV}$.

The EPIC spectra of NGC 7009 are clearly inconsistent with a power-law or blackbody spectrum. Thus, the X-ray emission from NGC 7009 must be thermal plasma emission and the spectral peak at $\sim 0.55 \mathrm{keV}$ corresponds to the He-like triplet of $\mathrm{O}$ VII at $\sim 0.57 \mathrm{keV}$. We can model the observed spectra in order to determine the physical conditions of the X-ray-emitting plasma and the amount of foreground absorption. We have adopted the thin plasma emission model of Raymond \& Smith (1977) and the nebular abundances determined from optical and UV spectra of NGC 7009. The abundances of He, C, N, $\mathrm{O}, \mathrm{Ne}$, and $\mathrm{S}$ relative to hydrogen by number have been measured to be $0.13,2.9 \times 10^{-4}, 2.2 \times 10^{-4}, 4.3 \times 10^{-4}$, 
$7.2 \times 10^{-5}$, and $1.9 \times 10^{-5}$, respectively (Kwitter \& Henry 1998). Accordingly, we have adopted nebular abundances, relative to the solar values (Anders \& Grevesse 1989), of $1.33,0.8,1.9,0.7,0.6$, and 1.2 for $\mathrm{He}, \mathrm{C}, \mathrm{N}, \mathrm{O}$, $\mathrm{Ne}$, and S, respectively, and 1.0 for the other elements. We have assumed solar abundances for the foreground interstellar absorption, and adopted absorption crosssections from Balucinska-Church \& McCammon (1992).

The spectral fits are carried out by folding the Raymond-Smith model spectrum through the corresponding EPIC/MOS1, EPIC/MOS2, and EPIC/pn response matrices, and fitting the modeled spectra to the observed EPIC spectra in the $0.3-1.5 \mathrm{keV}$ energy range using the $\chi^{2}$ statistics. The best-fit model, overplotted on the EPIC/pn and EPIC/MOS spectra in Fig. 2 (left), has a plasma temperature of $T=1.8 \times 10^{6} \mathrm{~K}$ (or $\left.k T=0.152 \mathrm{keV}\right)$, an absorption column density of $N_{\mathrm{H}}=8.1 \times 10^{20} \mathrm{~cm}^{-2}$, and a volume emission measure of $1.9 \times 10^{54} d^{2} \mathrm{~cm}^{-3}$ where $d$ is the distance to NGC 7009 in $\mathrm{kpc}^{1}$.

The goodness of the spectral fits is illustrated by the plot of reduced $\chi^{2}$ of the fits as a function of $k T$ and $N_{\mathrm{H}}$ shown in Fig. 2 (right). The $99 \%$ confidence contour spans $T=1.6-2.0 \times 10^{6} \mathrm{~K}($ or $k T=0.14-0.17 \mathrm{keV})$, $N_{\mathrm{H}}=2-17 \times 10^{20} \mathrm{~cm}^{-2}$, and volume emission measure $1.5-2.4 \times 10^{54} d^{2} \mathrm{~cm}^{-3}$. Assuming that the H I column density is similar to the absorption column density and adopting a typical gas-to-dust ratio (Bohlin et al. 1978), the corresponding visual extinction is $A_{V}=0.44_{-0.3}^{+0.5} \mathrm{mag}$, and the logarithmic extinction at the $\mathrm{H} \beta$ line is $c_{\mathrm{H} \beta}=$ $0.15_{-0.15}^{+0.22}$. This extinction is consistent with other estimates of extinction $\left(c_{\mathrm{H} \beta}=0.24 \pm 0.06\right.$, Lame \& Pogge $1996 ; c_{\mathrm{H} \beta}=0.10_{-0.08}^{+0.06}$, Rubin et al. 2001). The observed (absorbed) X-ray flux of NGC 7009 in the $0.3-1.5 \mathrm{keV}$ energy range is $7.2 \times 10^{-14}$ ergs $\mathrm{cm}^{-2} \mathrm{~s}^{-1}$, and the unabsorbed (intrinsic) X-ray flux is $1.7 \times 10^{-13} \mathrm{ergs} \mathrm{cm}^{-2} \mathrm{~s}^{-1}$. The total X-ray luminosity in the $0.3-1.5 \mathrm{keV}$ energy range is $L_{\mathrm{X}}=2.0 \times 10^{31} d^{2}$ ergs s${ }^{-1}=3 \times 10^{31}$ ergs s$^{-1}$ for a distance of $1.2 \mathrm{kpc}$.

\section{Discussion}

XMM-Newton EPIC observations of NGC7009 have shown that the diffuse X-ray emission is confined within the central cavity. The temperature of the X-ray-emitting gas, $1.8 \times 10^{6} \mathrm{~K}$, is below that expected for the shocked fast wind from its central star which has a terminal velocity $\sim 2800 \mathrm{~km} \mathrm{~s}^{-1}$ (Cerruti-Sola \& Perinotto 1989; Iping \& Sonneborn 2001). Similar situation is observed in the other PNe with diffuse X-ray emission: the plasma temperatures implied by the X-ray spectra of $\mathrm{BD}+30^{\circ} 3639$ and NGC 6543 are several times lower than the expected post-shock temperature for their fast stellar wind (Kastner et al. 2000; Chu et al. 2001). This temperature difference is expected if heat conduction is prevalent at the interface

1 The distance estimates to NGC 7009 range from 0.5 to $2.1 \mathrm{kpc}$. In the following we will adopt a distance of $1.2 \mathrm{kpc}$. between the shocked fast wind and the dense nebular shell (Soker 1994; Zhekov \& Perinotto 1996).

In order to determine the role played by the hot gas in the nebular dynamics of NGC 7009, it is possible to derive the rms density and pressure of the $\mathrm{X}$ ray-emitting gas and compare them to those of the surrounding cool nebular shell. Assuming a prolate ellipsoidal central cavity, the volume occupied by the X-ray-emitting gas is $\sim 6.7 \times 10^{51}(d / 1.2)^{3} \epsilon \mathrm{cm}^{3}$, where $\epsilon$ is the filling factor. From this volume and the aforementioned volume emission measure we derive an rms density of $20(d / 1.2)^{-1 / 2} \epsilon^{-1 / 2} \mathrm{~cm}^{-3}$, and a total mass for the $\mathrm{X}$ ray-emitting gas of $8 \times 10^{-5}(d / 1.2)^{5 / 2} \epsilon^{1 / 2} M_{\odot}$. This rms density and the plasma temperature of $1.8 \times 10^{6} \mathrm{~K}$ imply that the thermal pressure of the X-ray-emitting gas is $\sim 4 \times 10^{-9}(d / 1.2)^{-1 / 2} \epsilon^{-1 / 2}$ dynes $\mathrm{cm}^{-2}$. The nebular shell surrounding the hot gas has a density of $5000 \mathrm{~cm}^{-3}$, a temperature of $10500 \mathrm{~K}$ (Hyung \& Aller 1995; Rubin et al. 2001), and thus a thermal pressure of $\sim 10^{-8}$ dynes $\mathrm{cm}^{-2}$. The thermal pressure of the nebular shell is comparable or higher than that of the X-ray-emitting gas unless the filling factor $\epsilon$ is $\ll 0.2$.

The X-ray luminosity of NGC 7009 and the density and pressure of the hot gas in its interior are the lowest among the four PNe for wich diffuse X-ray emission has been detected thus far. These differences may be associated with the nebular evolution. NGC 7009 has a large nebular shell, $0.145 \times 0.06 \mathrm{pc}$ in size, and dynamical age, $\sim 1700 \mathrm{yrs}$ for an expansion velocity of $17 \mathrm{~km} \mathrm{~s}^{-1}$ (Bohigas et al. 1994). Therefore, it appears to be in the most advanced evolutionary stage among these four PNe. At a more advanced evolutionary stage, NGC 7293 does not have any detectable diffuse X-ray emission (Guerrero et al. 2001). The Chandra and XMM-Newton observations of these five $\mathrm{PNe}$ suggest that $\mathrm{PNe}$ are brightest in X-rays when they are young and NGC 7009 may be at an evolutionary stage when the diffuse X-ray emission starts to decline. This evolutionary effect is expected as the stellar wind diminishes and the central cavity grows. More observations of $\mathrm{PNe}$ at different evolutionary stages are needed to evaluate the duration of the pressure-driven phase in $\mathrm{PNe}$ and its effects on the nebular dynamics. As the evolved PNe are expected to have low X-ray surface brightness, the high sensitivity of XMM-Newton is essential in detecting such PNe.

Acknowledgements. We thank Steve Snowden for advice on the XMM-Newton data reduction, Guillermo García-Segura for enlightening discussion on the X-ray emission from $\mathrm{PNe}$, and Rosina Iping, George Sonneborn, and Robert H. Rubin for useful discussion on the physical properties of NGC 7009. We also thank the referee, Joel Kastner, for his constructive comments. This research was supported by the NASA grant NAG 5-10042.

\section{References}

Anders, E., \& Grevesse, N. 1989, Geochim. Cosmochim. Acta, 53, 197

Balucinska-Church, M., \& McCammon, D. 1992, ApJ, 400, 699 
Bohigas, J., López, J. A., \& Aguilar, L. 1994, A\&A, 291, 595

Bohlin, R. C., Savage, B. D., \& Drake, J. F. 1978, ApJ, 224, 132

Cerruti-Sola, M., \& Perinotto, M. 1989, ApJ, 345, 339

Chu, Y.-H., Guerrero, M. A., Gruendl, R. A., Williams, R. M., \& Kaler, J. B. 2001, ApJ, 553, L69

Dahlem, M. 1999, XMM Users' Handbook

Guerrero, M., Chu, Y.-H., \& Gruendl, R. A. 2000, ApJS, 129, 295

Guerrero, M. A., Chu, Y.-H., Gruendl, R. A., Williams, R. M., \& Kaler, J. B. 2001, ApJ, 553, L55

Hyung, S., \& Aller, L. H. 1995, MNRAS, 273, 973

Iping, R. C., \& Sonneborn, G. 2001, American Astronomical Society Meeting, 198, 1306
Kastner, J. H., Soker, N., Vrtilek, S. D., \& Dgani, R. 2000, ApJ, 545, L57

Kastner, J. H., Vrtilek, S. D., \& Soker, N. 2001, ApJ, 550, L189 Kwitter, K. B., \& Henry, R. B. C. 1998, ApJ, 493, 247

Kwok, S. 1983, in Planetary Nebulae, ed. D. R. Flower (Dordrecht: Reidel), IAU Symp., 103, 293

Lame, N. J., \& Pogge, R. W. 1996, AJ, 111, 2320

Raymond, J. C., \& Smith, B. W. 1977, ApJS, 35, 419

Rubin, R., et al. 2001, MNRAS, submitted

Soker, N. 1994, AJ, 107, 276

Strüder, L., Briel, U., Dennerl, K., et al. 2001, A\&A, 365, 18 Weaver, R., McCray, R., Castor, J., Shapiro, P., \& Moore, R. 1977, ApJ, 218, 377

Zhekov, S. A., \& Perinotto, M. 1996, A\&A, 309, 648 